\title{
Sleep Quality Assessment (PSQI)
}

\section{What is PSQI, and what is it measuring?}

The Pittsburgh Sleep Quality Index (PSQI) is an effective instrument used to measure the quality and patterns of sleep in adults. It differentiates "poor" from "good" sleep quality by measuring seven areas (components): subjective sleep quality, sleep latency, sleep duration, habitual sleep efficiency, sleep disturbances, use of sleeping medications, and daytime dysfunction over the last month.

\section{INSTRUCTIONS:}

The following questions relate to your usual sleep habits during the past month only. Your answers should indicate the most accurate reply for the majority of days and nights in the past month. Please answer all questions.

\section{During the past month,}

1. When have you usually gone to bed?

2. How long (in minutes) has it taken you to fall asleep each night?

3. What time have you usually gotten up in the morning?

4. A. How many hours of actual sleep did you get at night?

B. How many hours were you in bed?

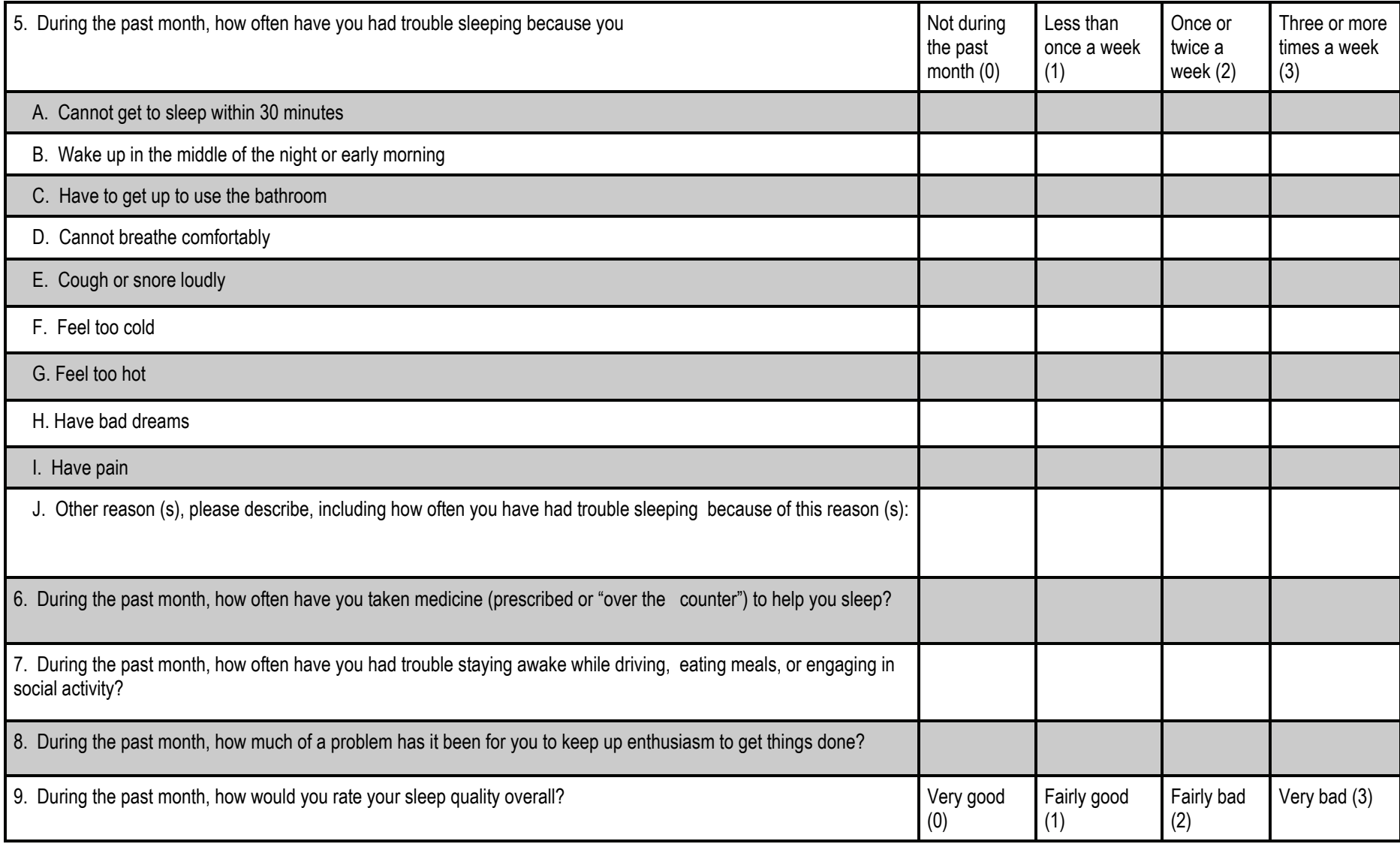

\section{Scoring}

\section{Component 1 \#9 Score}

Component 2 \#2 Score (<15min (0), 16-30min (1), 31-60 min (2), >60 min (3))

$+\# 5$ a Score (if sum is equal $0=0 ; 1-2=1 ; 3-4=2 ; 5-6=3$ )

Component 3 \#4 Score (>7(0), 6-7 (1), 5-6 (2), <5 (3)

Component 4 (total \# of hours asleep) / (total \# of hours in bed) $\times 100$

$>85 \%=0,75 \%-84 \%=!, 65 \%-74 \%=2,<65 \%=3$

Component 5 \# sum of scores $5 b$ to $5 j(0=0 ; 1-9=1 ; 10-18=2 ; 19-27=3)$

Component 6 \#6 Score

Component $7 \quad \# 7$ Score + \#8 score $(0=0 ; 1-2=1 ; 3-4=2 ; 5-6=3)$

C1

$\mathrm{C} 2$

$\mathrm{C} 3$

$\mathrm{C} 4$
$\mathrm{C} 5$
$\mathrm{C} 6$
$\mathrm{C} 7$

